\title{
TMAR: Extension of a Tabletop Interface using Mobile Augmented Reality *
}

\author{
Sewon $\mathrm{Na}^{1}$, Mark Billinghurst ${ }^{2}$, and Woontack Woo ${ }^{1}$ \\ ${ }^{1}$ GIST U-VR Lab. \\ Gwangju, 500-712, S. Korea \\ \{sna,wwoo\}@gist.ac.kr \\ ${ }^{2}$ The HITLabNZ, University of Canterbury, New Zealand \\ mark.billinghurst@hitlabnz.org
}

\begin{abstract}
Recently, many researchers have worked on tabletop systems. One issue with tabletop interfaces is how to control the table without using conventional desktop input devices such as a keyboard or mouse. A second issue is allowing multiple users to simultaneously share the tabletop system. In this paper we explore how Augmented/mixed reality (AR/MR) technology can be used to explore these issues. One advantage of AR technology is being able to bring $3 \mathrm{D}$ virtual objects into the real world without needing to use a desktop monitor and allows users to intuitively interact with the objects. In this paper we describe a Tabletop Mobile AR system that combines a tabletop and a mobile interface. The Tabletop system can recognize user gestures and objects and a user can intuitively manipulate and control it. In addition, multiple users have equal access for information on the table enabling them to easily share digital content. This makes possible unique entertainment and education applications. In this paper we describe the technology, sample entertainment interfaces and initial user feedback.
\end{abstract}

\section{Introduction}

In everyday life, tables provide space for entertainment, education and supporting meetings between multiple persons. For example, we sometimes play games around the table or talk with friends while having drinks on the table. In this case it forces people to concentrate on one point and it allows everyone equal access to the table.

Recently, many researchers have worked on electronic tabletop systems. One important issue of with tabletop systems is how to control the table without using conventional desktop input devices such as a keyboard or mouse. Some researchers have proposed input methods using graspable or tangible objects [1][13] while others have developed multi-touch technology [2]. For example, Microsoft's Surface computing project allows users to intuitively interact tabletop system by using multitouch technology. However, touch screen manufacturing is difficult and the bigger the screen is, the more expensive it is.

* This research was supported by the UCN Project, the MIC 21C Frontier R\&D Program in Korea. 


\section{Sewon Na1, Mark Billinghurst2, and Woontack Woo1}

In addition, other research has been conducted on how to enable multiple users to share tabletop interfaces simultaneously. Information displayed on the screen is usually shown for only one user from one direction, which limits concurrent accessibility from a variety of direction. The ability to sharing the table with multiple users allows users to access data significantly different way from desktop environments [14]. Some ways of doing this including splitting the table screen according to user number or using a spherical screen which can rotate left or right [3], [4]. However, the former is insufficient for accessibility in sharing and collaboration manner because each split screen view is still shown for one person. A spherical screen is also difficult because the users often continuously compete to get the authority of control. Thus it is difficult to ensure simultaneous control opportunity of the table.

In this paper, we present a tabletop mobile AR system in order to solve the problems mentioned above. Augmented/mixed reality (AR/MR) technology has been studied by many researchers in mobile and wearable computing fields to bring virtual 3D objects into real space without having any vertical monitor [15], [16]. In addition, AR interaction allows users to intuitively interact with the $3 \mathrm{D}$ virtual objects. The Tabletop-mobile AR system combines a tabletop interface with a mobile interface using such an AR. The tabletop interface consists of a rear-project, rear-camera and touch-frame. It allows convenient 2D interaction through recognizing hands and objects put onto the table without conventional input devices such as a keyboard or mouse. The mobile interface consists of an ultra mobile PC with a camera at rear and a screen being able to touch at front. This interface registers virtual contents with video image from camera and then provides methods for user to easily interact with virtual contents by using the touch-interaction. Thus, this interface allows the users to interact intuitively augmented 3D information by using a mobile device with attached camera as well as the table interaction.

The proposed system divides into two spaces as a common space and a personal space. It allows multiple users to collaborate between two spaces. The table interface plays a role as a collaborative space. Thus, It can support collaboration and sharing for multi-user. While, the use of personal mobile device ensures that anyone can easily accessibility the tabletop interface and unlike previous tabletop systems. It extend tabletop interface and get out of limitation of scope of tabletop screen. Moreover, Users can personally manipulate $3 \mathrm{D}$ virtual objects according to personalized information such as user's profile or preferences.

This paper is organized a six sections. Section 2 explains an initial prototype system called ARTable [13], which identified several problems with tabletop systems. While Section 3 explains a novel system we developed to overcome these problems. Section 4 explains how to implement the system. Finally we present conclusions in Section 5 .

\section{$2 \quad$ ARTable}

We briefly explain an earlier tabletop system we developed called ARTable, which identified several problems common to desktop interfaces. Our current research is 
based on this earlier work and overcomes these problems. The ARTable is consists of a tabletop display with a projector and camera underneath it connected to a computer (see Fig. 1(a)). The top of the table is a semi-transparent material so it can be projected onto and objects placed on it can be seen by the camera underneath. Using computer vision software [9] can recognize and track objects placed on the table.

Objects placed on the table have a square fidducial marker on their base, which can be detected by the camera under the table, and so the object position is known in table coordinates. For each object the $2 \mathrm{D}$ position and rotation relative to the table is found and so objects can be used to trigger events on the table. However, with rear-projector, rear-camera systems, there can be difficulty with marker detection due to projected imagery shown on the marker (see Fig.1 (b)). This makes it difficult for the marker tracking software to find the fidducial marker. One solution is to project a white circle around the object, enabling the tracking marker to be easily seen. However in order for this to work and initial object position must be known.

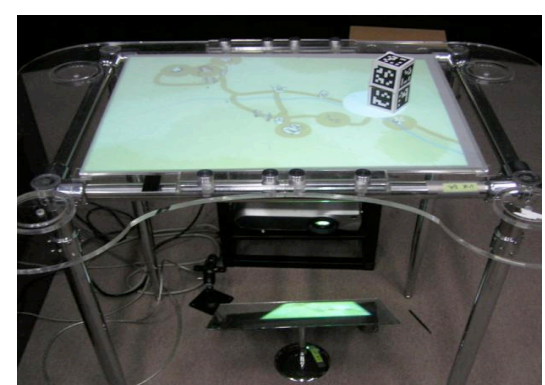

(a)

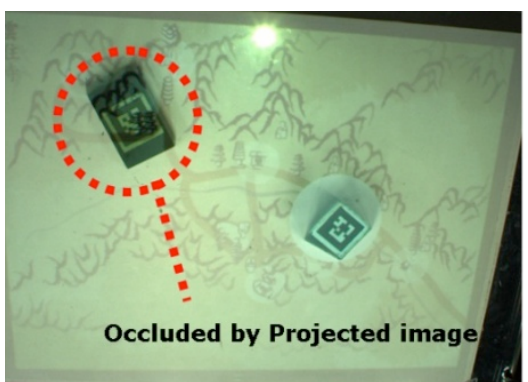

(b)

Fig. 1. ARTable (a) upper surface of the table (b) back surface of the table through underneath camera

There are two ways to find the initial object position and so change the projected imagery so that the object can be tracked. First, the system can make the entire screen white and waits until the first object is detected before projecting the application image. If the entire screen color is white, the objects placed on the table are not obscured by any projected images. Once the object is detected, the screen is restored to the desired image, and when the user gets rid of the object from table, the white screen again shown awaiting object detection. Although this is a very simple method, it has a critical problem in that there can be only one tracked object. After one object is detected, the table image is no longer white and further objects are obscured by the projected imagery.

A second method is to use additional device for finding the position of objects on the table. In our prototype we used the cost effective NextWindow touch frame [12] placed over the table. This uses cameras in the frame to touch enable any surface and so provides an initial position of any object placed on the table. We can use the coordinates given from the touch frame to provide the object position and enables us to project a white circle around the object. The camera under the table can then be 
used to find the object orientation. The advantage of this approach is that many objects can be tracked at once.

Our initial ARTable was used in a number of applications. For example, Dream of Mee-luck was interactive storytelling system on a Korean traditional tale, and ARTable was used as interaction tool for its virtual world navigation. [6]

However there were a number of limitations with this system. First users around the table did not have a view of the application aligned to their position, so the ARTable did not solve the view problem mentioned in section 1. Second, users could not see $3 \mathrm{D}$ virtual content and there was no intuitive support for 3D interaction. In the next section we describe our current tabletop mobile AR system, which overcomes both of these problems.

\section{Tabletop Mobile AR System}

\subsection{System overview}

Our tabletop mobile augmented reality (AR) system consists of two types of interfaces: a table interface (the previous ARTable) and a mobile interface (see Fig. 2). The table interface consists of the modules for input and output, and networking. The mobile interface also consists of the modules for input and output, and networking but it also adds a tracking module which use a camera attached to back of the mobile device.

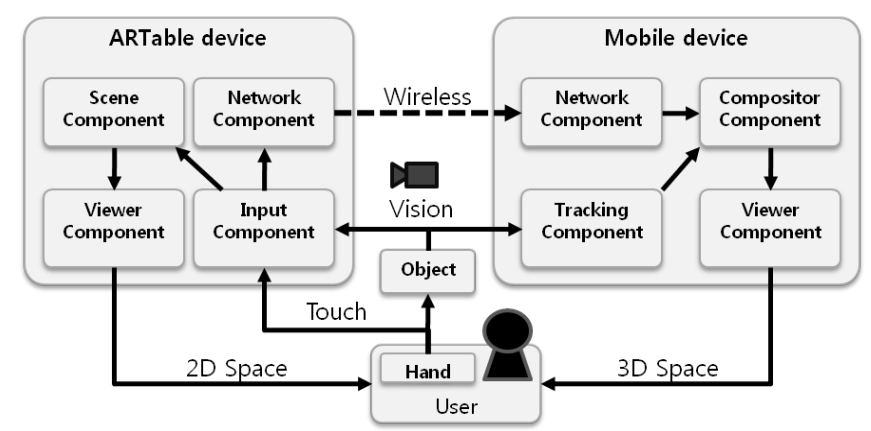

Fig. 2. The system diagram of the tabletop mobile AR system

The tabletop interface is a version of the ARTable previously describe. So the user can interact with projected images on the tabletop by using hand gestures or tangible objects in various ways. However, the mobile device allows the user to see 3D virtual images overlaid on live video from its camera through mobile device's screen. The user can also manipulate these 3D virtual images on the handheld device. 


\subsection{Table Interface (Collaborative interface)}

The tabletop interface plays a role as collaborative space. In this collaborative space, Multi-users can simultaneously manipulate virtual contents in table screen by using hand interaction. Also users can select the virtual contents through placing physical objects onto it exactly. (The use of Selected contents will explain in next section) The tabletop interface can recognize and track the user's hands or other objects using a camera under the table and NextWindow touch-frame as described in section 2 . The physical objects used are cardboard squares representing materials, selection tools or other control widgets. In the future, real symbolic objects such as a souvenir taken from travels could substitute these. The NextWindow touch-frame technology can only detect one object position at a time and so will not report the correct position of a second object once the first is placed on the table. We overcome this by raising the touch frame a centimeter off the table surface (see Fig. 3(a)). In this way when an object marker is placed on the table its position is detected when it is being placed or removed, but whiles on the table it sits below the touch frame and so does not interfere with the detection of other object positions. Therefore, Multi-users can place own physical objects simultaneously and table system connects it to the virtual content as one-by-one. Of course, once the object is on the table it can be robustly tracked by the camera under the table even though users move objects at the same time.

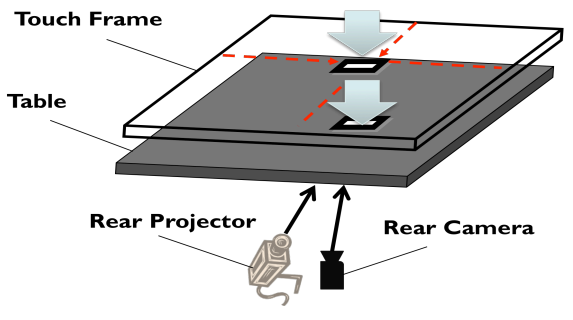

(a)

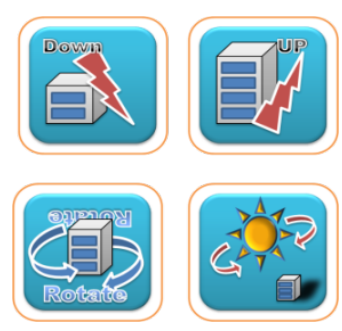

(b)

Fig. 3. Table system (a) concept of tabletop interface (b) the gap between touch-frame and the screen

An interaction method of tabletop system defines as button based interface to easily manipulate the contents. A sort of buttons consists of simply selective buttons and hand gesture recognition based buttons executing differently according to hand's movement. (See Fig. 3(b)) Simply selective button suits with discontinuous selection behavior such as creating or deleting. While a hand gesture based buttons suit with continuously changeable behavior such as position, scale and rotation change. 


\subsection{The Mobile AR Interface (Personalized interface)}

In our interface we use a handheld PC to increase accessibility to the table through the use of mobile AR technology. Using the mobile interface the user can interact in 3D space by using body position and orientation instead of conventional finger interaction on a screen. This allows extending the range of visual distance to users as well as supporting a private space. Moreover, it support for users to equivalent controllability in the mobile device wherever users are.

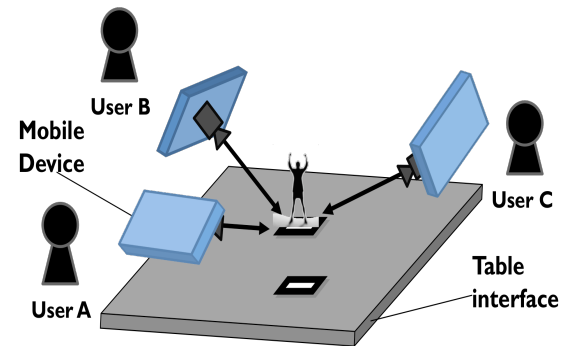

(a)

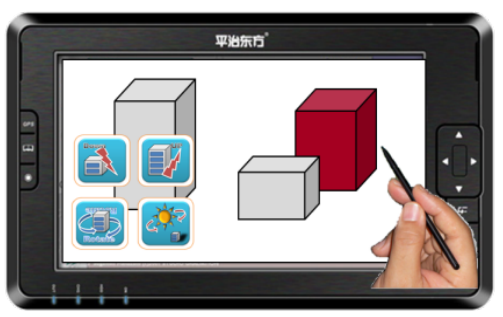

(b)

Fig. 4. Mobile Interface (a) sharing table using mobile device around the table (b) users with the handheld device around the tabletop interface

Object Tracking \& Augmentation. In order to track and recognize the objects placed on the table, objects on the table have tracking markers on their top and bottom. The handheld PC tracks the markers on the top using the ARToolkitPlus tracking library [9]. This identifies the specific object being tracked and determines the pose of the handheld PC relative to the tracked objects. In this way 3D virtual imagery can be overlaid on the real objects and viewed through the handheld PC in real time. In the Mobile AR interface we use a Tangible AR user interface metaphor [20] to interact between the table and the mobile device. By using one tangible object as an input object, the users can see 3D virtual objects superimposed over live video on the screen of the mobile display device. (See Fig. 4(a))

Interaction. In the mobile $\mathrm{AR}$, the user will interact with $3 \mathrm{D}$ Contents. So, we basically can think selecting and manipulating 3D contents. User can explicitly select 3D objects through touch panel on the mobile interface as well as on the table. Because touch panel is 2D surface, we use ray-tracing methodology to select 3D contents. Ray-tracing algorithm is usually used in the computer graphics area. In addition, user can select implicitly objects placed at the center of mobile devices without any selection because it is trivial that object seen through mobile device is user's interest object. Practically, user carry mobile device on one hand, the hand is not free. So we use button interaction method to manipulate the objects. If the object is selected, mobile device shows buttons about actions with respect to the object and user can easily select or manipulate through pushing buttons. (See Fig. 4(b)) 


\subsection{Seamless connection between Collaborative and Personalized interface (Common Space)}

One of our goals is to ensure that the users feel as through the two interfaces (tabletop, mobile AR) support seamless interaction in one common space on and above the table. In order to achieve this goal, we have to correctly align the two spatial coordinates systems, and provide temporal synchronization and unified data sharing. Aligning the spatial coordinates can be done through the placing reference objects on the table. As shown in Fig 5(a), we can obtain absolute 3D coordinates by the combination of relative 3D coordinates of the object in the mobile AR devices frame of reference and 2D Absolute coordinates of objects put on the table. ' $\mathrm{W}$ ' in the figure represents an obtained absolute coordinate result. ' $U$ ' represents 2D Absolute coordinate of table. ' $\mathrm{V}$ ' is a $3 \mathrm{D}$ coordinate in the mobile AR devices. When two devices transfer the coordinate data through networking, we conduct temporal synchronization to prevent data overflow or congestion by latency. Thus, we make pipe for data serialization as first-in first-out (FIFO) and control data (see Fig.5b). This structure waste out-of-time data and reduce unnecessary data.

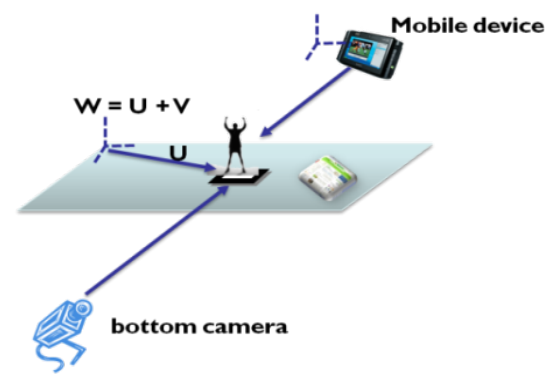

(a)

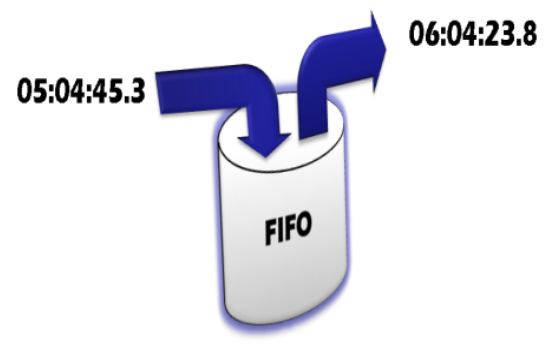

(b)

Fig. 5. Constructing common space (a) spatial coordinates correction (b) temporal synchronization

\section{Implementation}

\subsection{System setup}

We have implemented a prototype tabletop mobile AR interface using the following hardware and software. First of all, the table interface consists of glass with semi transparent tracing paper attached and the NextWindow touch-frame [12] mounted on top. Under the table, there is a NEC short throw projector [21] and Dragonfly camera [10] connected to workstation PC. Secondly, the mobile AR interface was based on a Sony VAIO ultra mobile PC [21] with an embedded USB camera on the back. The 
two computers are connected with wireless Ethernet networking. The software running on the tabletop interface was based on the OpenSceneGraph [7] library, while on the handheld PC we used osgART Library [8]. The projected table view was based on the osgViewer module of OSG and all virtual objects are represented as SceneGraph objects. On the handheld device through the use of osgART library, we can easily represent augment reality areas combined with virtual world and computer vision. In addition, we used Point Grey Library to capture images [10]. To communicate between two interfaces, we employed TAO library, which follows the CORBA specifications, based on ACE [11].

\subsection{TMAR system}

The TMAR system mainly consists of two interfaces: the tabletop interface and the mobile AR interface. The tabletop interface uses two types of input devices from the user. First one is tracking hands by using touch frame as we explained above. The user can intuitively select 3D contents or buttons (see Fig. 6(a)). The second one is recognizing and tracking physical objects on the table by underneath camera. The user usually put the physical objects on the 3D contents of the table screen (see Fig. 6(b)). It plays a role of carriers allowing coupling of both interfaces (mobile/tabletop) seamlessly.

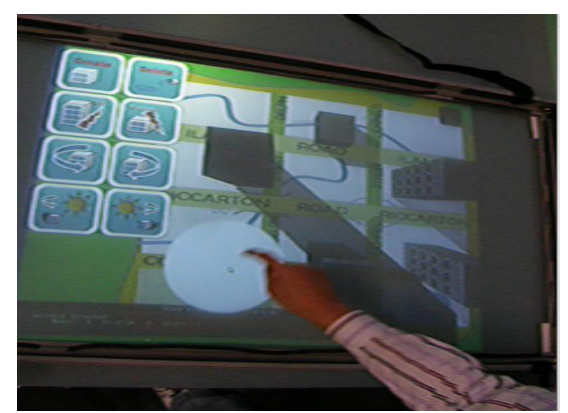

(a)

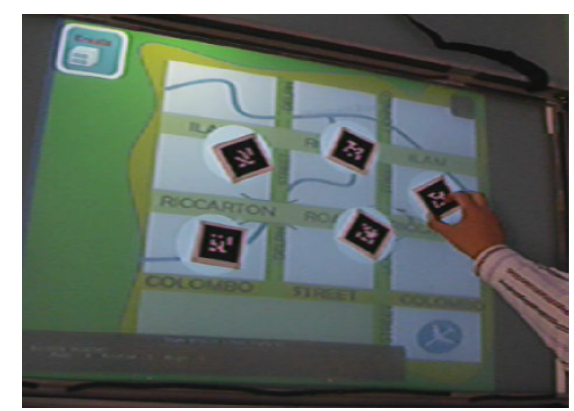

(b)

Fig. 6. The tabletop interface (a) tabletop interface recognizing hand gesture (the white circle is a clicked position) (b) physical objects on the table

While the mobile interface uses touch panel input device. Thus, the user can touch the panel on the handheld device and select 3D contents. 3D contents are displayed on the physical objects as one by one with it. The user can see registered image with video image (see Fig. 7(a)). Fig. 7(b) shows that the user selects 3D content (a scribed building represent selected one) and then buttons, which are applicable, become displayed below it. As push the button, the user can rotate buildings (see Fig. 7(c)) or raise it (see Fig. 7(d)). In Fig 7(d), it multiple 3D contents are selected and then they are modified at once. Also the user can create or delete the contents (see Fig. 7(e)). 


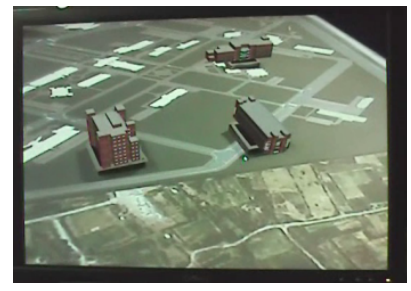

(a)

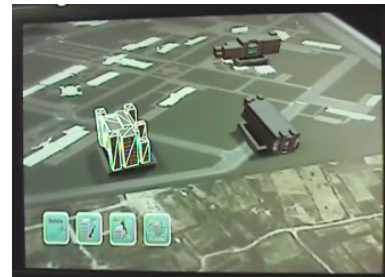

(b)

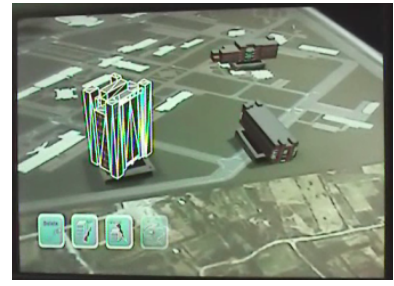

(c)

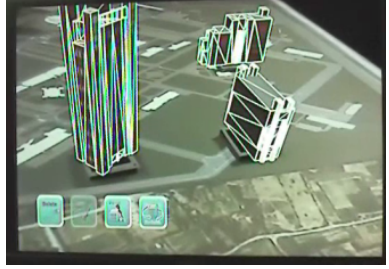

(d)

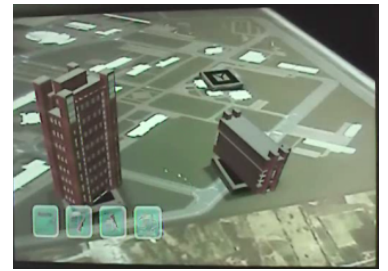

(e)

Fig. 7. Mobile AR interface views and Interactions (a) Augmented buildings on the markers (b) Selection and showing button (c) rotating a building (d) raising buildings (e) deleting a building

\subsection{Urban Planning Application}

The prototype interface described above could be used in many possible applications. We are particularly interested in how this could facilitate the development of new types of edutainment applications. Our first test application was for an urban planning task (see Fig. 8). Urban planning is to plan the city's structure or landscapes by architects. This application has been utilized in AR area [18], [19]. We made simple map consist of $3 \times 2$ roads and 9 sites (shown in Fig. 8a). Users can create buildings on it or manipulate through tabletop interface (shown in Fig. 8(b), 8(c)). Also it is possible to see and interact through mobile AR interface (shown in Fig. 8(d)). 


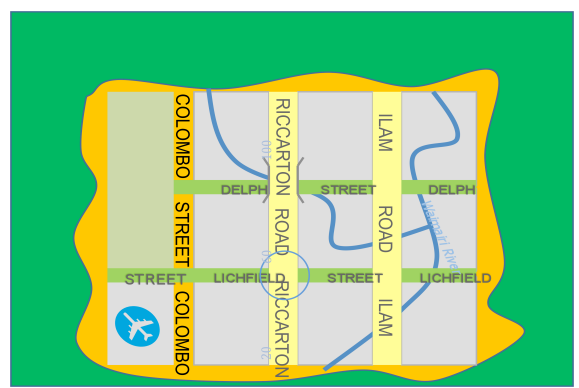

(a)

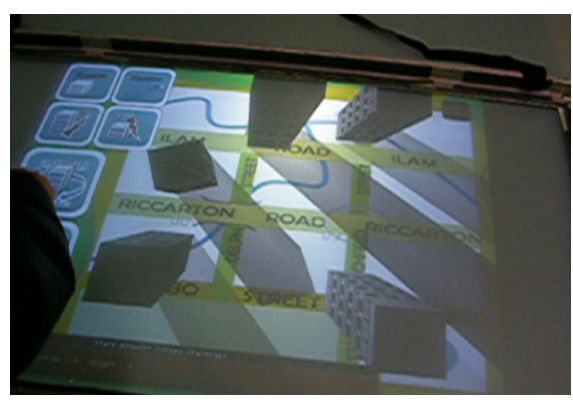

(c)

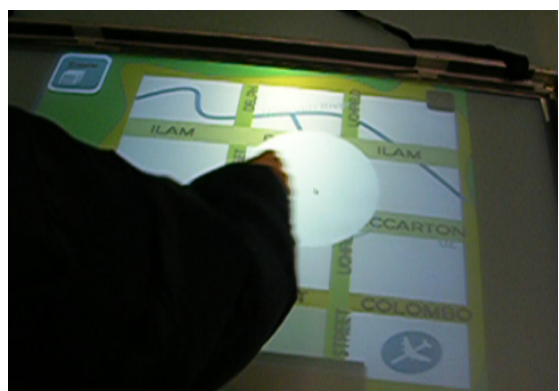

(b)

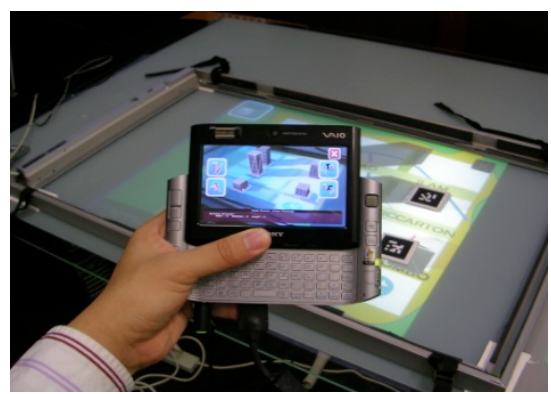

(d)

Fig. 8. Tabletop Mobile AR system (a) the simple map for urban planning scenario (b) the tabletop interface with the map (c) building manipulation in the tabletop interface (d) urban planning through the mobile AR interface

\subsection{User Study}

To asset usability of the proposed system, we have conducted a simple user study with the prototype application. The experiment goal is to conduct an urban planning project. Each person is tested 3 kinds of system with that goal. First, they use fingers to touch the screen instead of mouse or keyboard. Second, they use some markers and a mobile device to achieve the same goal. Lastly, they will combine the first and second methods.

The user study procedure is like followings. We give them few minutes to freely place 5 buildings. They may use any buttons to make a city that they want, but there is one rule to remember when they are constructing the city: shadows should never overlap. Thus, we are going to be able to compare 3 systems through the task completion time. Currently, we continuously are testing people with the scenario. Through a preliminary observation, we realize some users confused using TabletopMobile interface because there are two kinds of interfaces. For example, in order to move objects on the table, user should look at the table. On the other hand, in order to manipulate virtual objects, user should look at the mobile. Thus, if such an 
interchanging process is frequently occurred, users found it difficult. Finally, we are trying to solve this problem. We will complement our system and enhance it.

\section{Conclusion and Future work}

In this paper we have presented a prototype tabletop mobile AR interface, which supports AR Interaction between virtual and physical objects. The Tabletop interface can recognize user's hand and objects and intuitively manipulate and control them. The advantage and novelty of our research is that multiple users had equal access for information on the table enabling them to easily share digital content. This made possible unique entertainment and education applications. In the future, each module is integrated and executed on one mobile device. We are going to study more about mobile collaboration in complex situation. The users may want to reflect a result of their manipulation to only the selected user. According to authority or position, the user's manipulation ability can be differentiated among users. For example, engineers of urban planning system have more power than consumers. Moreover, even if users see same buildings, they may want to see different building or landscapes according to their preference. Therefore, it is necessary to personalize the mobile AR interface. We believe that the proposed system is applicable for both education and entertainment.

\section{References}

1. Ullmer, B., Ishii, H.: The metaDESK: models and prototypes for tangible user interfaces. In Proceedings of the 10th annual ACM symposium on User interface software and technology, (1997) 223-232

2. Han, J. Y.: Low-Cost Multi-Touch Sensing through Frustrated Total Internal Reflection. In Proceedings of the 18th Annual ACM Symposium on User Interface Software and Technology, (2005) 115-118

3.Shen, C.,Vernier, F.D.,Forlines, C.,Ringel, M.: DiamondSpin: An Extensible Toolkit for Around-the-Table Interaction, ACM Conference on Human Factors in Computing Systems (CHI), (2004) 167-174

4. Scott, S.D., Carpendale, M.S.T., and Inkpen, K.: Territoriality in Collaborative Tabletop Workspaces. Proceedings of CSCW, (2004) 294-303

5. http://www.microsoft.com/surface/

6. Lee. Y., Kim, D., Lim, Y., Kim, K., Kim, H., and Woo, W.: Dream of Mee-luck: Aspiration for a New Dawn," LNCS (ICVS), vol.3805, (2005) 282-285

7. http://www.openscenegraph.org/projects/osg

8. http://www.artoolworks.com/community/osgart/

9. http://studierstube.icg.tu-graz.ac.at/handheld_ar/artoolkitplus.php

10. http://www.ptgrey.com/

11. http://www.cs.wustl.edu/ schmidt/ACE.html

12. http://www.nextwindow.com/

13. Park, Y., Woo, W.: The ARTable: An AR-based Tangible User Interface System, LNCS, vol. 3942, (2006) 1198-1207

14. Carsten M., Timo E., Daniel H. G.: A component based architecture for distributed, pervasive gaming applications. Advances in Computer Entertainment Technology (2006) 
15. S. Güven, S. Feiner,: Authoring 3D Hypermedia for Wearable Augmented and Virtual Reality, Proc. ISWC '03 (Seventh International Symposium on Wearable Computers), White Plains, NY, (2003) 118-226.

16. Azuma, Ronald T.: A Survey of Augmented Reality, Presence: Teleoperators and Virtual Environments 6, 4, (1997) 355-385.

17. Scott, S., Sheelagh, M., Carpendale, T., Inkpen, K.: Tabletop design: Territoriality in collaborative tabletop workspaces, Proceedings of the 2004 ACM conference on Computer supported cooperative work, ACM Press, (2004)

18. Buchmann. V., Violich. S., Billigust. M., Cockburn. A.: FingARtips: gesture based direct manipulation in Augmented Reality, In Proceedings of the 2nd international conference on Computer graphics and interactive techniques in Australasia and South East Asia, (2004) 212-221

19. Broll. W., Stoerring. M., Mottram. C.: The Augmented Round Table - a new Interface to Urban Planning and Architectural Design, Ninth IFIP TC13 International Conference on Human-Computer Interaction, (2003) 1103-1104

20. Ishii, H., Ullmer, B.: Tangible bits: towards seamless interfaces between people, bits and atoms, In Proceedings of the SIGCHI conference on Human factors in computing systems, (1997) 234-241

21. Http://www.nec.co.jp/techrep/en/journal/g06/n03/060319.html 